\title{
Immunomodulatory effects and anti-Candida activity of lactobacilli in macrophages and in invertebrate model of Galleria mellonella
}

\author{
Felipe Eduardo de Oliveira a , Rodnei Dennis Rossoni ${ }^{\text {a, * }}$, Patricia Pimentel de Barros a, \\ Barbara Evelyn Begnini ${ }^{a}$, Juliana Campos Junqueira a , Antonio Olavo Cardoso Jorge a, \\ Mariella Vieira Pereira Leão ${ }^{\mathrm{b}}$, Luciane Dias de Oliveira ${ }^{\mathrm{a}}$ \\ ${ }^{a}$ Department of Biosciences and Oral Diagnosis, São Paulo State University (Unesp), Institute of Science and Technology, São José dos Campos, Avenida \\ Engenheiro Francisco José Longo 777, São Dimas, CEP: 12245-000, São Paulo, Brazil \\ ${ }^{\mathrm{b}}$ Institute of Basic Biosciences, School of Medicine, University of Taubaté, Av. Tiradentes, 500, Centro, Taubaté, CEP: 12030-180, São Paulo, Brazil
}

\section{A R T I C L E I N F O}

\section{Article history:}

Received 11 May 2017

Received in revised form

17 July 2017

Accepted 7 August 2017

Available online 8 August 2017

\section{Keywords:}

Probiotic

Lactic acid bacteria

Lactobacillus

Yeasts

Macrophages

Mycology

\begin{abstract}
A B S T R A C T
Due to the growing number of multi-resistant Candida spp., adjuvant treatments that may help combat these fungal pathogens are relevant and useful. This study evaluated the immunomodulation and antiCandida activity of Lactobacillus rhamnosus (LR), Lactobacillus acidophilus and Lactobacillus paracasei suspensions, either single- or multiple-strain, in mouse macrophages (RAW 264.7) and Galleria mellonella (GM). Mouse macrophages were activated by different lactobacilli suspensions and challenged with C. albicans (CA). Tumor necrosis factor (TNF)- $\alpha$, interleukin IL-1 $\beta$, IL-6 and IL-17 production and cell viability were investigated. LR was the best suspension for stimulating all evaluated cytokines and thus was used in subsequent in vivo assays. Two C. albicans clinical strains, CA21 and CA60, were then added to the GM assays to further confirm the results. LR suspension was injected into the larvae $24 \mathrm{~h}$ before challenging with CA. Survival curve, CFU per larva and hemocytes were counted. In the GM, the LR suspension increased the survival rate and hemocyte counts and decreased the CFU per larva counts for all groups. Lactobacilli suspensions presented strain-dependent immunomodulation; however, single suspensions showed better results. Anti-Candida activity was demonstrated by decreased Candida counts in the GM with the use of LR.
\end{abstract}

(C) 2017 Elsevier Ltd. All rights reserved.

\section{Introduction}

Candida yeasts are the primary agents of fungal infection in patients who are immunosuppressed from the use of immunosuppressive drugs, advanced age and systemic diseases [1-3]. Over $90 \%$ of all opportunistic mycoses are caused by Candida species [4-7]. Since fungi invade tissues, they are subjected to opsonization in which phagocytes are activated by receptors and intracellular signals for phagocytosis [8]. These include the standard receptors for host cell recognition, such as C-type lectin receptors and Tolllike receptors $[9,10]$. In healthy, immunocompetent individuals,

\footnotetext{
* Corresponding author.

E-mail addresses: felipe.eoliveira@ymail.com (F.E. de Oliveira), dennisrossoni@ hotmail.com (R.D. Rossoni), barrosdnapp@yahoo.com.br (P.P. de Barros), ba. begnini@gmail.com (B.E. Begnini), juliana@fosjc.unesp.br (J.C. Junqueira), olavojorge@fosjc.unesp.br (A.O.C. Jorge), mariellavieira@yahoo.com.br (M.V.P. Leão), luciane@ict.unesp.br (L.D. de Oliveira).
}

the innate immune system (neutrophils and macrophages) provides protection against fungal species through phagocytosis and cytokines such as interleukin (IL)-1 $\beta$, IL-6, IL-17 and tumor necrosis factor (TNF)- $\alpha$, which are important in targeting immune responses to fungi $[11,12]$.

T-lymphocytes (T-cells) are integral to the host's adaptive immune response to $C$. albicans infection and provide direct and indirect means of controlling fungal proliferation. Th17 cells release cytokines, including IL-17 and IL-22, and are critical for immune protection against $C$. albicans in most mucosal sites. In addition, Th17 cells are regarded as the predominant cells for protecting against $C$. albicans in oral and dermal candidiasis [13,14]. Th17 differentiation is influenced by IL-6, which is produced by epithelial cells in response to $C$. albicans infections [15]. Notably, the production of both IL- 6 and IL-23 by antigen presenting cells results from recognition of mannan in C. albicans [14].

The frequency of invasive fungal infections and resistance to antifungal therapy continue to increase despite the introduction of 
new antifungal agents; therefore, the emergence of resistant fungal strains is a growing concern [16]. Due to the need for alternative anti-fungal methods, probiotics offer a potential resolution. Probiotics are involved in modulating the immune response by increasing nonspecific phagocytic activity through macrophage activation [17,18], altering the release of pro- and antiinflammatory cytokines $[19,20]$, competing with pathogens for nutrients and receptors, preventing the harmful effects of pathogens and stimulating the release of bacteriocins, hydrogen peroxide and lactase [21,22]. Moreover, probiotic suspensions can be constituted by a single species or by the association of two or more species [19-21], and their beneficial effects are related to the strain and dose [22].

In vitro and in vivo studies are necessary to better understand the interaction among hosts, pathogens and probiotics. In vitro studies are used to elucidate the role of probiotics [19,23-25], and they represent an important step in this field. For in vivo studies, invertebrate models are used [26,27] to investigate fungal pathogenicity and the role of probiotics. An advantage of this model is that it meets the ethical and legal requirements of the 3 Rs (reduction, refinement and replacement) in addition to being an alternative to mammalian use [28,29].

Although there are many probiotic studies in the literature, it is necessary to examine the effects of different probiotic suspensions, isolated or combined, on the immune response to fungal infections. Different strains can induce different responses, and their interactions may influence the immune response and, consequently, the type of immunomodulation provided. This study evaluated the production of TNF- $\alpha$, IL-1 $\beta$, IL- 6 and IL-17 in macrophages activated by different lactobacilli suspensions and challenged by $C$. albicans to verify the immunomodulatory role of probiotic bacteria on a G. mellonella model.

\section{Materials and methods}

\subsection{Macrophage cell culture}

RAW 264.7 macrophages were obtained from the cell bank of the Paul Ehrlich Technical Scientific Association (APABCAM, Rio de Janeiro, Rio de Janeiro). These cells were grown in tissue culture flasks with DMEM culture medium (Dulbecco's Modified Eagle Sigma-Aldrich, St. Louis, MO, USA) supplemented with $10 \%$ fetal bovine serum (complete medium) and incubated at $37{ }^{\circ} \mathrm{C}$ at an atmospheric humidity of $5 \% \mathrm{CO}_{2}$.

\subsection{Microorganisms}

Initially, C. albicans (CA) ATCC 18804 and 3 species of Lactobacillus: L. rhamnosus (LR) ATCC 7469, L. acidophilus (LA) LA 14 and L. paracasei (LP) 19.1 were used in this study. All fungal strains and LR were from the Laboratory of Microbiology and Immunology of the Institute of Science and Technology of São José dos Campos/ UNESP (São Paulo State University, São José dos Campos, São Paulo, Brazil). LA was isolated from a commercial product (Prolive-Ache ${ }^{\circledR}$, Guarulhos, São Paulo, Brazil) and LP was isolated from the human oral cavity of an individual without tooth decay, per the approval of the Ethics Committee under protocol 560.479.

Lactobacilli strains were grown on Man-Rogosa-Shape agar (Himedia, Mumbai, India) for 4 days at $37{ }^{\circ} \mathrm{C}$ under microaerophilic conditions $\left(5 \% \mathrm{CO}_{2}\right)$. Isolated colonies were seeded in MRS broth. To prepare the suspensions, the species cultures were centrifuged for $5 \mathrm{~min}$ at $8300 \times \mathrm{g}$ separately, and the supernatant was discarded. The sediments were resuspended in sterile and pyrogen-free $0.9 \%$ $\mathrm{NaCl}$ and centrifuged twice. After centrifugation, the pellet was resuspended to a ratio of 25:1 (lactobacilli cells:macrophages) [30] and standardized in a spectrophotometer with a wavelength of $540 \mathrm{~nm}$ and optical density of 0.4 . Three monospecies (LR, LA and LP) and four multispecies (LR + LA, LR + LP, LA + LP and LR + LA + LP) suspensions were obtained. The multispecies suspensions were obtained by combining the monospecies suspensions in equal parts.

The $C$. albicans suspension was prepared from overnight cultures in $5 \mathrm{~mL}$ of yeast-nitrogen base (YNB) broth (Difco Laboratories Inc, Detroit, MI, USA) at $37{ }^{\circ} \mathrm{C}$ for $24 \mathrm{~h}$. The cells were then centrifuged at $2.000 \times \mathrm{g}$ for $10 \mathrm{~min}$ and the supernatant was discarded. The sediment was resuspended in sterile and pyrogen-free $0.9 \% \mathrm{NaCl}$ and mixed in shaker tubes for $30 \mathrm{~s}$. Cell washing was repeated twice. Fungal cells were adjusted to a ratio of 2:1 (fungal cells:macrophages) using a hemocytometer.

\subsection{Selection of the best lactobacillus strain to stimulate cytokines: In vitro study}

\subsubsection{Macrophage activation}

Macrophages were distributed into 24-well polystyrene microplates $\left(5 \times 10^{5}\right.$ viable cells per well) with DMEM supplemented with $10 \%$ fetal bovine serum to a final volume of $1 \mathrm{~mL}$. After $24 \mathrm{~h}$ of incubation $\left(37{ }^{\circ} \mathrm{C}\right.$ and $5 \% \mathrm{CO}_{2}$ ) for cell adhesion, the wells were washed three times with pyrogen-free, sterile saline to remove nonadherent cells. Aliquots of $500 \mu \mathrm{L}$ of DMEM and $500 \mu \mathrm{L}$ of pyrogen-free $0.9 \% \mathrm{NaCl}$ with either the different lactobacilli suspensions or pyrogen-free $0.9 \% \mathrm{NaCl}$ (control group) were added to each well, resulting in a ratio of 25:1 $\left(125 \times 10^{5}\right.$ lactobacilli: $5 \times 10^{5}$ macrophages). The plates were incubated at $37{ }^{\circ} \mathrm{C}$ in $5 \% \mathrm{CO}_{2}$ for $2.5 \mathrm{~h}$. After incubation, the wells were washed three times with pyrogen-free, sterile $0.9 \% \mathrm{NaCl}$ to remove lactobacilli. Next, $500 \mu \mathrm{L}$ of DMEM with streptomycin $\left(2 \% \mathrm{v.v}^{-1}\right)\left(\mathrm{Gibco}^{\circledR}\right.$, São Paulo, Brazil) and $500 \mu \mathrm{L}$ of pyrogen-free $0.9 \% \mathrm{NaCl}$ with either $C$. albicans or pyrogen-free $0.9 \% \mathrm{NaCl}$ (control group) were added to each well, resulting in a ratio of 2:1 (fungal cells:macrophages). The plates were again incubated at $37{ }^{\circ} \mathrm{C}$ in $5 \% \mathrm{CO}_{2}$ for $2.5 \mathrm{~h}$.

After incubation, the plates were washed three times with pyrogen-free, sterile saline to remove microbial cells, and $1 \mathrm{~mL}$ of DMEM with streptomycin and amphotericin B $\left(1 \%{\left.\mathrm{v} . \mathrm{v}^{-1}\right)}^{-1}\left(\mathrm{Gibco}^{\circledR}\right.\right.$, São Paulo, Brazil) was added. The plates were incubated $37^{\circ} \mathrm{C}$ in $5 \%$ $\mathrm{CO}_{2}$ for $16 \mathrm{~h}$ to release the cytokines, and the supernatants were collected and maintained at $-80{ }^{\circ} \mathrm{C}$ to quantify the cytokines.

\subsubsection{Cytokine quantification}

A commercial ELISA kit (R\&D Systems, Minneapolis, MN, USA) was used to determine the concentration of TNF- $\alpha$, IL-1 $1 \beta$, IL- 6 and IL-17 per the manufacturer's instructions. The optical density for the cytokine quantification tests was measured using a microplate reader (EL808 - BioTek Instruments Inc., Winooski, Vermont, USA).

\subsubsection{Cell viability assay}

This experiment verified whether macrophage viability would be affected by probiotics or C. albicans. Macrophages were distributed into 96-well polystyrene microplates at a ratio of $25: 1\left(1 \times 10^{6}\right.$ lactobacilli: $4 \times 10^{4}$ macrophages) with DMEM to a final volume of $100 \mu \mathrm{L}$. The rest of the procedure was performed as previously described in section 2.3.1, maintaining the same ratios and incubation periods.

After the $16 \mathrm{~h}$ incubation period, the wells were washed and the test was performed per the manufacturer's instructions (Biotool, Houston, Texas, USA). The microplates were read in a spectrophotometer (Cambrex Elx808cse - Lonza, Basel, Switzerland) at a wavelength of $570 \mathrm{~nm}$, and the optical densities were converted into a viability percentage.

It is important to note that at the end of the $16 \mathrm{~h}$ incubation 
period, all microbial species were dead due to the streptomycin and amphotericin B in the medium, removing any possibility of a falsepositive result in the macrophage viability. In addition, three washes were performed between each step of the protocol to remove any microbial cells left in the wells.

\subsubsection{Selection of the lactobacilli suspension}

The best lactobacilli suspension, either in combination or as a single species, for $C$. albicans was chosen based on the results of the cytokine and viability assays. The probiotic suspension used to conduct the in vivo tests was chosen from the suspensions containing only one species. This was because the concentrations were the same in all suspensions for both the combined species and the single species $\left(125 \times 10^{5}\right.$ viable cells per well in the macrophage activation assay and $1 \times 10^{6}$ viable cells per well in the cell viability assay).

\subsection{Lactobacillus rhamnosus interference in different strains of C. albicans: in vivo study}

\subsubsection{Susceptibility of Galleria mellonella to lactobacilli suspensions}

We determined the sub-lethal inoculum concentration of Lactobacillus by injecting $G$. mellonella larvae with serial dilutions of the probiotic bacteria. Different concentrations of each Lactobacillus strain $\left(10^{4}\right.$ to $10^{7}$ cells/larvae) were inoculated into the larvae through the last left proleg. The larvae were kept on petri dishes at $37^{\circ} \mathrm{C}$, and they were monitored daily for survival.

\subsubsection{Survival of G. mellonella after inoculation with L. rhamnosus} suspension and being challenged by different $C$. albicans strains

To determine the survival curve of $G$. mellonella, $L$. rhamnosus cells were injected into the last right proleg of the larvae. Twentyfour hours after inoculation with $L$. rhamnosus cells, $C$. albicans cells were injected into the last left proleg of the larvae.

Two other $C$. albicans clinical strains were added to the GM assays to further confirm the results: C. albicans 21 (CA21) and C. albicans 60 (CA60). Both strains were from the Laboratory of Microbiology of the Institute of Science and Technology of São José dos Campos/UNESP (São Paulo State University) and were isolated from oropharyngeal candidiasis lesions of HIV-positive patients from Emílio Ribas Infectology Institute (Instituto de Infectologia Emílio Ribas, São Paulo, Brazil), with the approval of the Ethics Committee (Protocol 051/2009-PH/CEP). The samples were cultured in chromogenic HiCrome Candida medium (Himedia, Mumbai, India), identified by biochemical methods (API20C System- BioMérieux, Paris, France) and confirmed via molecular methods (PCR Multiplex) according Junqueira et al. [31].

C. albicans inocula were prepared by growing $50 \mathrm{~mL}$ YPD cultures overnight at $30^{\circ} \mathrm{C}$. Cells were pelleted at $1.300 \times \mathrm{g}$ for $10 \mathrm{~min}$ followed by three washes in PBS. Cell densities were determined by hemocytometer count. Sixteen randomly chosen G. mellonella larvae $(330 \pm 25 \mathrm{mg})$ were infected with $10 \mu \mathrm{L}$ of a suspension containing Candida in $10^{6} \mathrm{CFU}$ per larva in the last right proleg with a Hamilton syringe. In brief, G. mellonella larvae in the final instar larval stage were stored in the dark and used within 7 days from the date of shipment. Before injection, the area was cleaned using an alcohol swab, and after injection, larvae were incubated in petri dishes $\left(37^{\circ} \mathrm{C}\right)$. The number of dead $\mathrm{G}$. mellonella was scored every $24 \mathrm{~h}$ for 7 days. Larvae were considered dead when they did not respond to touch.

\subsubsection{CFU per larva counts in the G. mellonella hemolymph}

To quantify the presence of $C$. albicans in the infected G. mellonella, experiments were conducted on the euthanized larvae at 12 and $24 \mathrm{~h}$ post-infection. The experimental groups were divided by the interaction of $L$. rhamnosus with each $C$. albicans strain, as well as the control group with each $C$. albicans strain. The experiment was performed in triplicate.

Each larva was sectioned with a scalpel in the cephalo-caudal direction and compressed to remove the hemolymph, which was placed in a microtube. Serial dilutions $\left(10^{-1}\right.$ and $\left.10^{-2}\right)$ were made from the hemolymph and seeded in petri dishes containing Sabouraud dextrose agar with chloramphenicol, a culture medium selective for $C$. albicans. The plates were incubated at $37^{\circ} \mathrm{C}$ for $48 \mathrm{~h}$, and the colonies were counted to calculate the $\mathrm{CFU} / \mathrm{mL}^{-1}$.

\subsubsection{Hemocyte density}

Larvae were infected by injecting $C$. albicans at the last left proleg $24 \mathrm{~h}$ after the $L$. rhamnosus was injected at the last right proleg. Hemocytes were collected from the hemocoel at $24 \mathrm{~h}$ post-
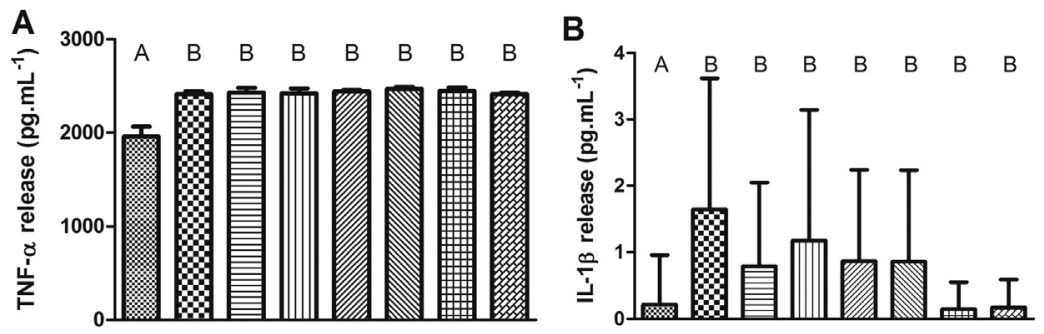

D

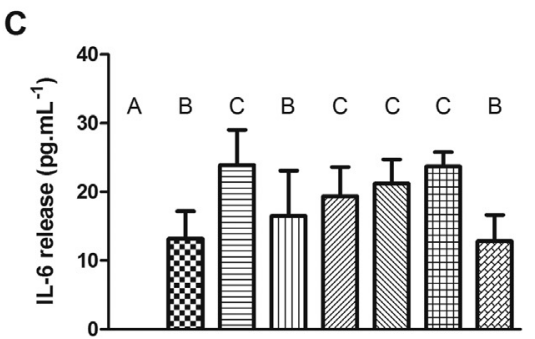

D

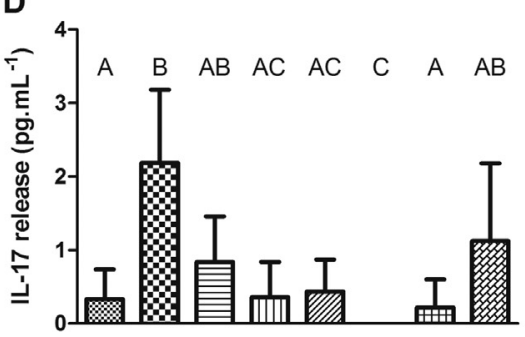

CA control

Ca CA+LR

$\mathrm{CA}+\mathrm{LA}$

ㄸ. $C A+L P$

m. $C A+L R+L A$

$C A+L R+L P$

CA+LA+LP

$C A+L R+L A+L P$
$A: p<0.0001$

$B: p=0.0135$

C: $p<0.0001$

D: $p<0.0001$

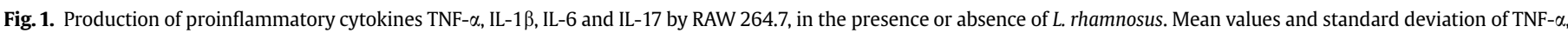

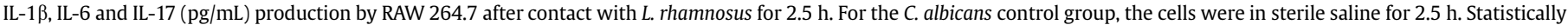

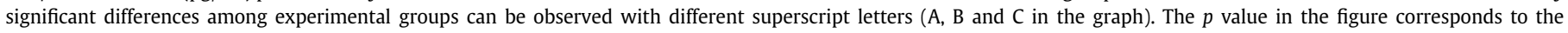
comparison of all groups. ANOVA and Tukey Test, $p=0.05$. 
Table 1

Cell viability, expressed in percentage, according to the lactobacilli suspension and fungal strain.

\begin{tabular}{lll}
\hline Cell Viability (\%) & CA & Sterile saline \\
\hline LR & 102.94 & 109.42 \\
LA & 95.64 & 95.55 \\
LP & 96.84 & 109.09 \\
LR + LA & 99.83 & 98.25 \\
LR + LP & 100.95 & 92.32 \\
LA + LP & 93.60 & 89.78 \\
LR + LA + LP & 91.98 & 103.03 \\
Negative control & 97.65 & 100 \\
\hline
\end{tabular}

$\mathrm{CA}=$ C. albicans; $\mathrm{LR}=$ L. rhamnosus; $\mathrm{LA}=$ L. acidophilus; $\mathrm{LP}=$ L. paracasei.

injection with C. albicans. Four larvae were bled into four tubes per group, containing cold sterile insect physiologic saline (IPS) (150 mM sodium chloride; $5 \mathrm{mM}$ potassium chloride; $100 \mathrm{mM}$ Tris-hydrochloride, pH 6.9 with $10 \mathrm{mM}$ EDTA and $30 \mathrm{mM}$ sodium citrate). The hemocytes were counted using a hemocytometer. We did not differentiate between the six types of hemocytes, and the results were averaged from three replicates.

\subsection{Statistical analysis}

Cytokine counts were analyzed by a one-way ANOVA and Tukey's test when the results passed a normality test. When they failed to pass normality, Kruskal-Wallis and Dunn's tests were used. Killing curves were plotted, and statistical analysis was performed by the log-rank (MantelCox) test. Student's t-test was used to hemocyte densities and CFU per larva assays. Statistical analysis was performed using the software GraphPad Prism 5 (GraphPad Software, Inc., California, CA, USA). A P value $<0.05$ was considered significant.

\section{Results}

All lactobacillus suspensions stimulated macrophages to produce more TNF- $\alpha$ than the $C$. albicans control group (Fig. 1A). There was no difference between the different suspensions of probiotics tested ( $p=0.0460)$, but all suspensions stimulated more TNF- $\alpha$ production than the control group $(p<0.0001)$. The IL- $1 \beta$ production results were strain-dependent, although $L$. rhamnosus was able to stimulate more cytokine production $\left(1.64 \pm 1.97 \mathrm{pg} \mathrm{mL}^{-1}\right)$ than the other probiotic groups as well as the Candida control group $(p=0.0283$ ) (Fig. 1B). The macrophages challenged by C. albicans produced significantly more IL- 6 in all lactobacilli suspensions, both mono and multispecies ( $p<0.0001$ ) (Fig. 1C). The best lactobacilli suspension for stimulating IL-17 production was L. rhamnosus $\left(2.18 \pm 0.99 \mathrm{pg} / \mathrm{mL}^{1}\right)$ when compared with the other probiotic groups $(\mathrm{p}<0.0001)$ as well as the Candida control group $(p<0.0001)$ (Fig. 1D). This cytokine plays a key role in protecting mammals from fungal infections.

Macrophage cell viability is expressed as percentages in Table 1. The negative control group, which did not receive any microorganisms, was considered $100 \%$ viable. Cell viability was not affected by the treatments, since no group showed cell viability lower than $70 \%$.

Based on the in vitro results, the best lactobacilli suspension against $C$. albicans was L. rhamnosus; therefore, this suspension was used in the subsequent in vivo assays. First, we determined the sublethal concentration for G. mellonella by inoculating it with L. rhamnosus ATCC 7469, since no previous studies have been reported on this strain in a G. mellonella model. Standard suspensions of $L$. rhamnosus at a $10^{4}$ to $10^{7}$ cells/larva concentration were inoculated into G. mellonella, and survival curves were constructed (Fig. 2). The results showed that only concentrations greater than $10^{6}$ cells/larva were pathogenic to G. mellonella, since none of the larvae died at this concentration. Therefore, the concentration of $10^{6}$ cells/larva was adopted for all subsequent assays, since it is the same concentration as that used for $C$. albicans infections.

We then extended our in vivo assays to laboratory reference strain, C. albicans ATCC 18804, and two clinical isolates, CA21 and CA60, as previous studies demonstrated that these isolates exhibited intra-species variability in relation to pathogenicity in animal models [32,33]. In addition, we could exclude the hypothesis that the probiotic would be strain specific.

After the prophylactic treatment with $L$. rhamnosus and infection with the $C$. albicans strains, the survival curve of the larvae was evaluated. There was a statistically significant difference for all Candida strains tested between the following: L. rhamnosus + C. albicans ATCC 18804 and PBS + C. albicans ATCC 18804 control group $(p=0.0001)$; L. rhamnosus $+C$. albicans 21 and PBS + C. albicans 21 control group $(p=0.0008)$ and $L$. rhamnosus + C. albicans 60 and PBS $+C$. albicans 60 control group $(p=0.0008)$ (Fig. 3). Per these results, the survival rates increased by 68, 81 and $75 \%$ for the groups infected with C. albicans ATCC 18804, C. albicans 21 and $C$. albicans 60, respectively.

Additionally, we investigated the ability of L. rhamnosus to reduce the fungal load in the hemolymph of $G$. mellonella by counting the $C$. albicans CFU per larva. In the groups infected with C. albicans ATCC 18804, there was a statistically significant difference between the group treated with $L$. rhamnosus and the untreated group at both $12 \mathrm{~h}(p=0.0099)$ and $24 \mathrm{~h}(p<0.0001)$, with reductions of $0.93 \log$ and $1.41 \log$ in the CFU per larva

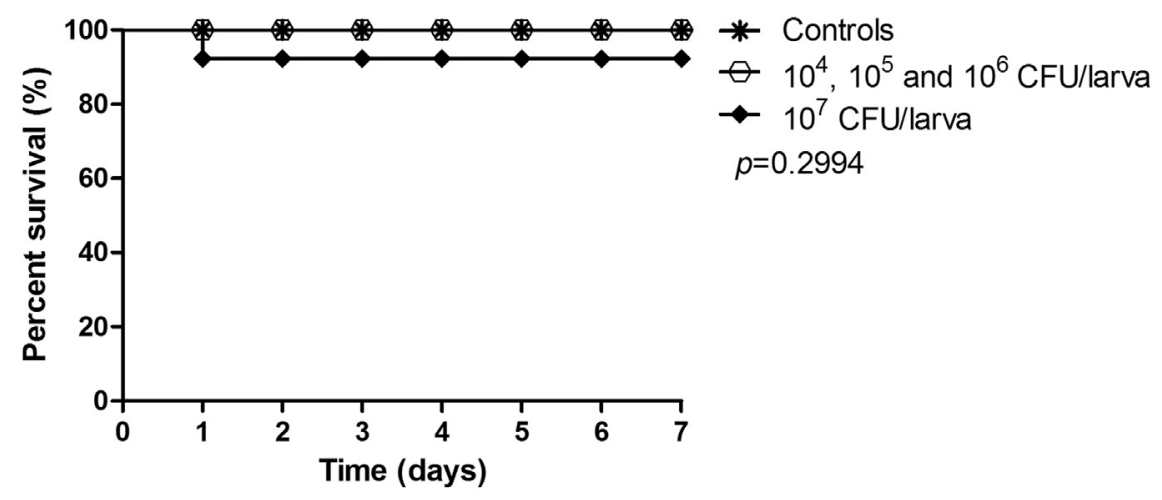

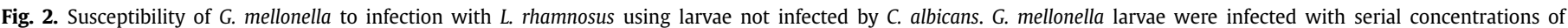

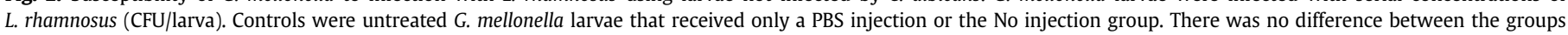
$(p=0.2994)$. Log-rank test, $p \leq 0.05$. 
A

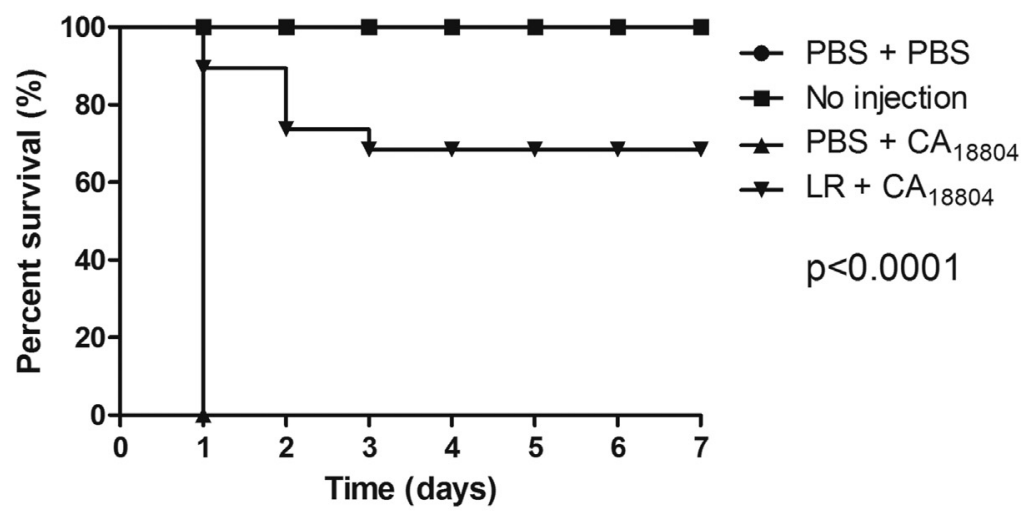

B

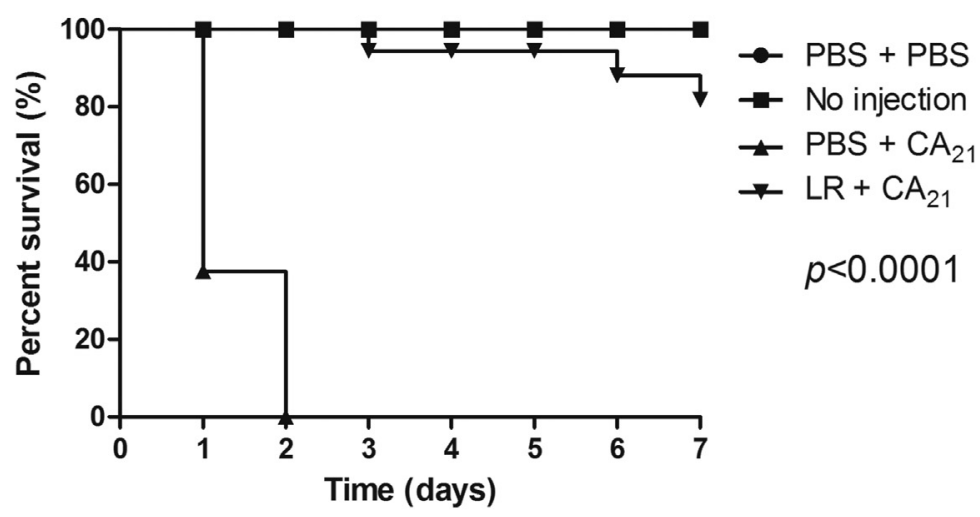

C

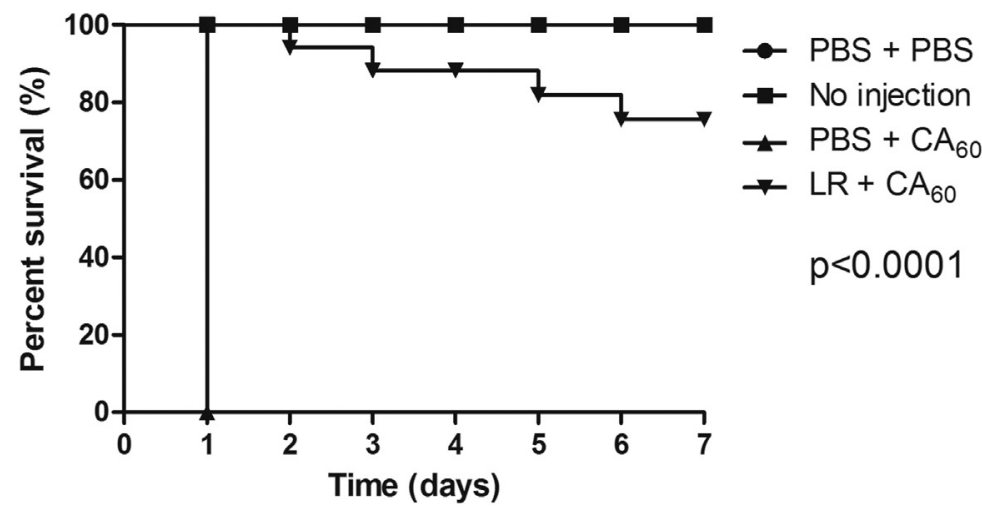

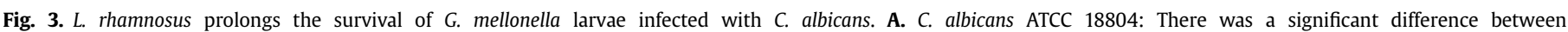

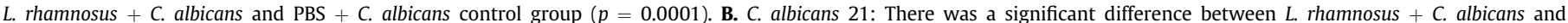

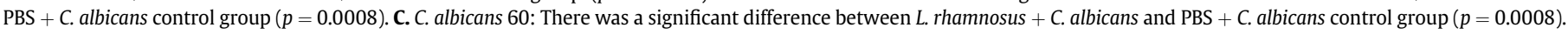
Log-rank test, $p=0.05$.

counts, respectively (Fig. 4A). For the groups infected with the clinical strains, there was a statistically significant difference only at $24 \mathrm{~h}$ for $C$. albicans $21(p=0.0023)$ and $C$. albicans 60 $(p=0.0001)$ and reductions of $1.49 \log$ and $1.85 \log$, respectively (Fig. 4B and C).

In the G. mellonella hemocyte counts, a statistical difference was obtained for all groups $(p<0.05)$. Prophylactic treatment with $L$. rhamnosus increased the number of hemocytes in all groups infected with the fungal strains as well as in the negative control group (L. rhamnosus + PBS), indicating that this Lactobacillus strain recruited hemocytes into the hemolymph. For the groups treated with $L$. rhamnosus and infected with $C$. albicans ATCC 18804 , there was a 2.15 -fold increase in the number of hemocytes. The same was observed for the groups infected with C. albicans 21 (4.26-fold increase) and C. albicans 60 (2.89-fold increase) and for the control group inoculated with PBS instead of the fungal strains (1.5-fold-increase). The means and the $p$ value for the comparison between groups are shown in Fig. 5. These results agreed with the survival assay and the CFU count assay, indicating that prior exposure to L. rhamnosus activates the G. mellonella immune system, which may allow the larvae to combat a lethal infection by $C$. albicans. 
A

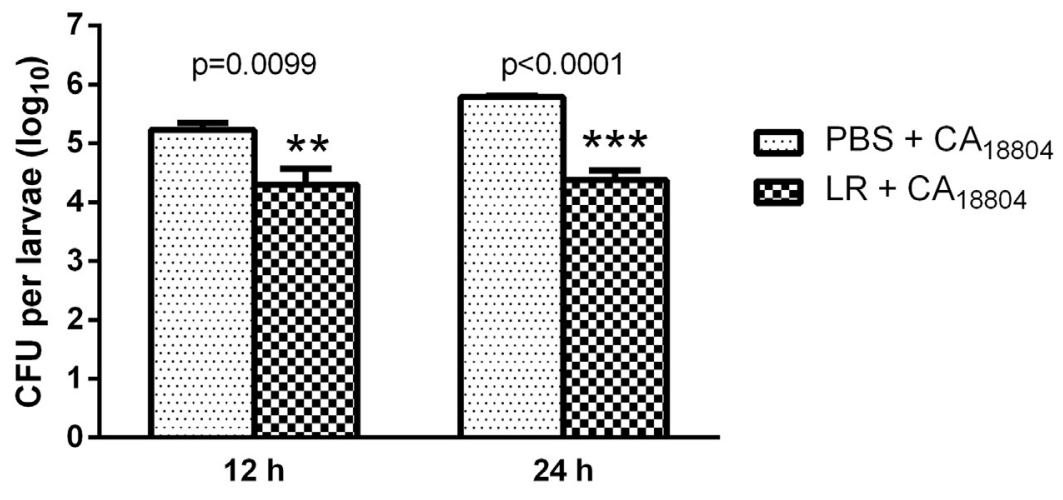

B

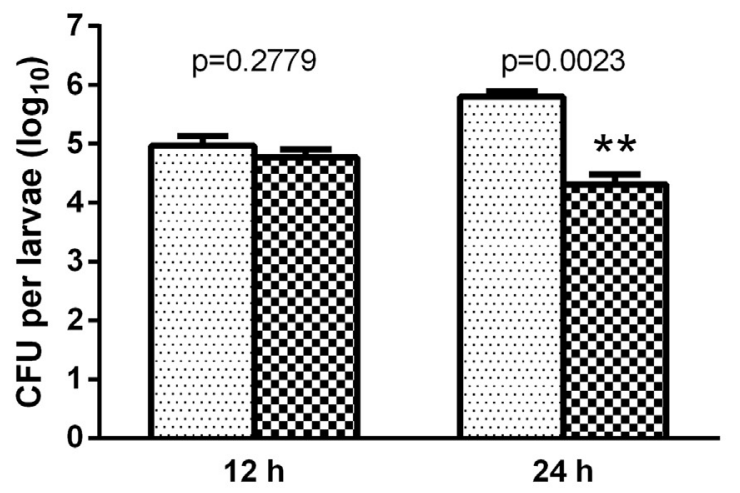

C

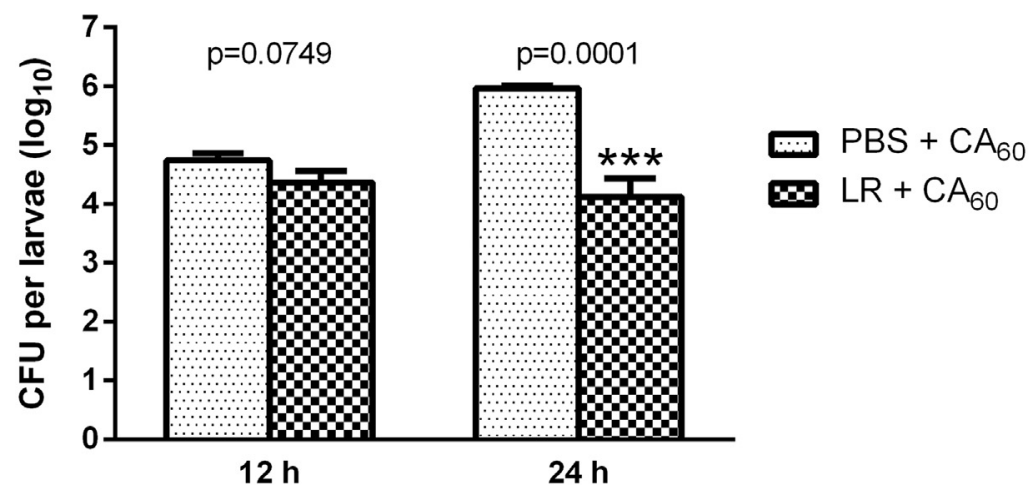

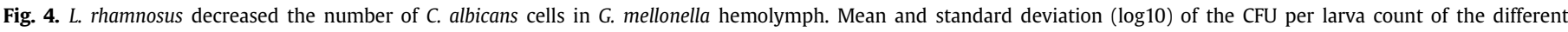

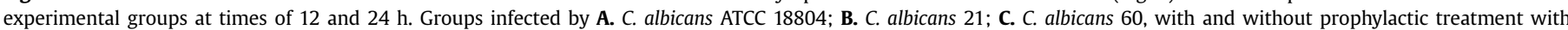

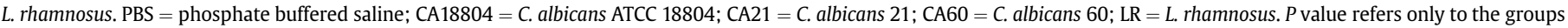
infected with Candida. Student's $t$-test, $\mathrm{p} \leq 0.05$

\section{Discussion}

Alternative treatments against resistant pathogens are gaining increasing attention in the scientific field. Among the most studied treatments, is the use of probiotics [27,34]. Probiotics are commensal microorganisms that benefit their host by providing anti-infective [35] and immunomodulatory properties [36,37].

In this study, the tests performed using mouse macrophages (RAW 264.7) directly reflect the central role of the innate immune system's defense mechanisms, including the inflammatory response [38] that produces chemical mediators such as cytokines, which are essential for host defense [39]. After activation and infection of the macrophages, their viability was verified to validate the other tests, as a high rate of cell death would make the results suspect. According to Li et al. [40], 70\% is the standard rate for determining whether the treatment is cytotoxic or not to the macrophages. Considering this, the treatments did not interfere with the macrophage viability.

In this study, there were statistically significant differences in cytokine production, and these differences varied by the species of lactobacilli in the suspension. Some authors have previously described lactobacilli modulation as being controversial, since it may increase, decrease or cause no change in cytokine levels $[41,42]$.

Romani [12] found that virulent strains of Cryptococcus neoformans can inhibit TNF- $\alpha$ production and induce IL-10 production, thus inhibiting macrophage activation. Although different fungal species were used in this study, we noted this low cytokine 


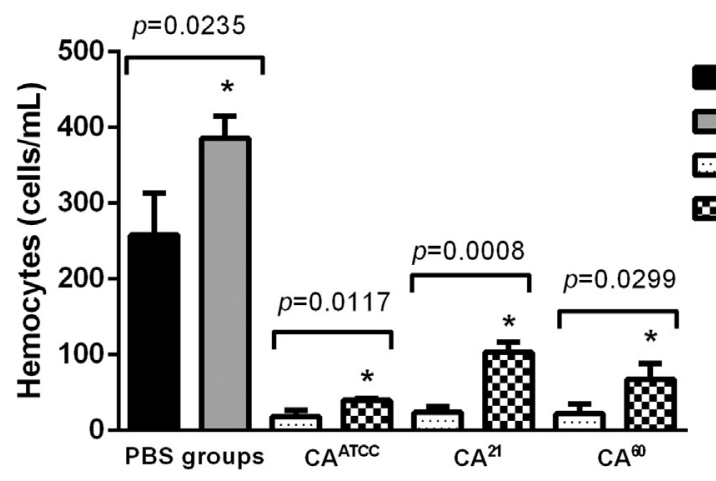

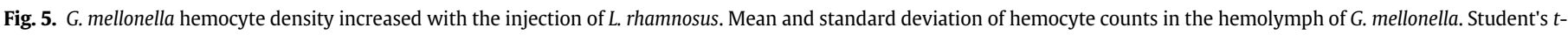
test was used to compare hemocyte densities between the experimental groups. A $p$ value $\leq 0.05$ was considered significant.

production when macrophages were only challenged with Candida. However, in the groups previously treated with lactobacilli suspensions, there was an increase in TNF- $\alpha$ production, indicating the immunomodulatory effect of the suspensions toward this cytokine.

Vonk et al. [43] and Hise et al. [44] showed that IL-1 $\beta$-deficient rats had a reduced survival and an increased fungal load when compared with healthy rats, showing the importance of this cytokine in host defense against fungal infections. In our results, the groups infected only with Candida presented low IL-1 $\beta$ production. When the macrophages were previously treated with lactobacilli suspensions, these averages either increased or remained similar in most cases, based on the fungal strain and lactobacilli suspension used. Therefore, the stimulation of IL- $1 \beta$ production provided by lactobacilli suspensions may play an essential role against fungal infection, since this cytokine is crucial for host defense in response to infection and injury [45].

Interleukin-6 levels in the Candida-infected cells were modulated when treated with lactobacilli, with a significant increase in all groups. IL-6 has a multifunctional role [46], mediating the direction of the inflammatory response, including the mechanisms of transition from innate to acquired immune response $[47,48]$. Genetic deletion of the of IL- 6 production mechanisms in mice was examined in 1994 [49], and the deletion resulted in an impaired response to infections, defects in the production of T-cell regulated antibodies and an absence of the acute phase response after infection or trauma. This suggests that IL- 6 production is important for the control of fungal infections, as well as serving as a signal to coordinate macrophages. In our study, the results showed that all lactobacillus suspensions are capable of increasing IL-6 production, an important aspect, since the group infected only by Candida showed very low production of this cytokine in response to the Candida.

We also analyzed the levels of IL-17 produced by macrophages. The results showed that in all experimental groups, only the group treated prophylactically with $L$. rhamnosus showed a statistically significant increase in IL-17 production. These results are promising for treatment against Candida infections, since $C$. albicans is the most pathogenic of the genus, and IL-17 plays an important role in innate and adaptive responses against infections at the mucosal and cutaneous interfaces. This was exemplified by the occurrence of chronic mucocutaneous candidiasis, in patients with genetic defects that impaired IL-17-related immune responses [50]. In addition, in patients treated with IL-17 antagonists, a higher incidence rate of Candida infections is expected [51]. These recent studies show the importance of finding a new alternative to improve the host defenses without inducing the emergence of resistant strains.

A cellular immune response against fungi is essential, and macrophages are the most important cells in the control of fungal growth [52]. When macrophages, challenged only by Candida, were evaluated in this study, the concentration of cytokines was lower than those in the groups previously treated with probiotics, indicating a low inflammatory mediator release profile. This suggests the pathogen's ability to escape recognition by the immune system and not activate host defense mechanisms.

Another important aspect is the composition of the microbial wall of lactobacilli. Lipoteichoic acid (LTA) and peptidoglycan are important components of the Gram-positive bacterial cell wall, capable of stimulating an immune response. However, the ability to influence responses is strain-dependent, since LTA and peptidoglycan may undergo species-dependent modifications [53-55]. Modifications in these structures may reflect different patterns of cytokine modulation.

The best lactobacilli suspension with immunomodulatory effects against $C$. albicans was $L$. rhamnosus (single species), and the study of this suspension was extended to in vivo assays using a G. mellonella model. In this study, the single Lactobacillus strain suspension produced better results than the multi-strain suspension. Agreeing with our results, MacPherson et al. [56] evaluated the immune modulation of TLR3-mediated inflammation in intestinal epithelial cells with single and multi-strain probiotic combinations, and they did not find statistically significant differences between the groups for either TNF- $\alpha$ or IL-8. In addition, IL- $1 \beta$ and IL-6 revealed no detectable protein modulation for all probiotic combinations, suggesting that the success of the probiotic combination varies with the strain studied.

Insects, such as $G$. mellonella larvae, possess only an innate immune system, which comprises humoral and cellular branches. Cellular response involves hemocytes, whose main function is phagocytosis of foreign bodies or sequestration of these bodies in the insect body in structures called nodules. As part of this humoral response, insects synthesize antimicrobial peptides, small molecules with bactericidal and fungicidal activity, by destabilizing the microbial membranes [57,58].

However, new studies have been changing this paradigm by suggesting that invertebrate immune responses are more complex and specific than previously thought. Primary exposure of invertebrates to pathogens increases their resistance to subsequent pathogenic challenges [59]. This mechanism has been reported in other invertebrate models $[60,61]$. Our results corroborate those of the previous studies, although the microorganism injected first cannot be considered pathogenic, since it did not affect the survival rate of the larvae in any of the tested concentrations. Moreover, previous studies have shown that the results derived from G. mellonella assays were positively correlated with results from studies with Caenorhabditis elegans and mice, demonstrating the 
potential that this invertebrate model has as an alternative to in vivo studies of probiotics strains [62-64].

In all survival assays, our study found statistically significant differences between the groups treated prophylactically with L. rhamnosus and those not treated. The lactobacilli suspension significantly increased the survival rate of the larvae infected with C. albicans $(\mathrm{p}<0.05)$ in all cases, suggesting that $L$. rhamnosus ATCC 7469 is a modulator in the immune response. Our study evaluated the survival of G. mellonella larvae similarly to Vilela et al. [26], Grounta et al. [65] and Ribeiro et al. [27], who prophylactically treated the larvae with Lactobacillus prior to the injection of fungal or bacterial pathogens. In all studies, they observed an increase in the larval survival rate that varied by strain and type of infection.

CFU counts for the G. mellonella hemolymph showed a decrease in the fungal load for all the strains evaluated when the larvae received prophylactic treatment with $L$. rhamnosus, in all time periods. However, we obtained statistically significant reductions in all groups, ranging from 96.2 to $98.6 \%$, only after 24 h. Our results are similar to those of other authors. Ribeiro et al. [27] found a reduction of $52.2 \%$ in the CFU count in the C. albicans ATCC 18804 biofilm with the addition of $L$. rhamnosus ATCC 9595 cells. Vilela et al. [26] verified a reduction in the G. mellonella hemolymph fungal load in the groups treated with L. acidophilus ATCC 4356 cells only at $24 \mathrm{~h}$ in accordance with our results. The result for the $12-\mathrm{h}$ period in the group infected by C. albicans ATCC 18804 differs from those found by Vilela et al. [26]. In our study, there was a significant reduction in the fungal load of $C$. albicans ATCC 18804 in the $12-\mathrm{h}$ time period. It is possible that this difference is due to the use of different strains of lactobacilli. As previously stated, modulation of the immune response is strain-dependent, i.e., it depends on both the pathogen and lactobacilli strains used.

Recently, members of our research group developed a study to evaluate the immunomodulatory action of the strain $L$. paracase $i$ 28.4 in experimental candidiasis in G. mellonella. Rossoni et al. [66] showed that prior exposure to L. paracasei 28.4 activates the G. mellonella immune system, which may allow the larvae to combat a lethal infection by $C$. albicans. This effect was mediated by an increase of circulating hemocytes and the production of elevated levels of antimicrobial peptides that consequently reduced Candida cells in G. mellonella hemolymph. These data demonstrate the need to invest in the strains used in this study as potential probiotics.

To understand the survival curve and CFU counts, a hemocytecounting assay was performed with the infected larvae, which plays a key role in the defense against fungal pathogens. Hemocyte density and survival of Galleria are indicators of pathogenicity $[67,68]$. Non-pathogenic fungi result in high hemocyte densities, while pathogenic fungi reduce hemocyte levels [68]. Our results demonstrate the modulatory power of the immune response of the L. rhamnosus suspension used. In all prophylactically treated groups, there was a significant increase in hemocyte density.

Density and survival of Galleria were related because they indicate fungal pathogenicity, which was verified by our results. For example, the groups treated with $L$. rhamnosus and infected with C. albicans ATCC 18804, had the lowest increase in survival, at approximately $37.5 \%$, and the lowest increase in hemocyte density (2.15-fold). It was the opposite for the clinical strains. The increase in survival may be related to the increased hemocyte density, confirming our results.

The results of this study corroborate other G. mellonella studies in the literature while simultaneously indicating the need for further studies using different assays (phagocytosis, production of phenoloxidase and antimicrobial peptides) to elucidate how the immunomodulation of probiotics acts on the host and eliminates pathogens.

\section{Conclusion}

Based on these results, Lactobacilli suspensions showed straindependent immune modulation, and single-strain suspensions showed better results than multiple-strain suspensions. L. rhamnosus ATCC 7469 showed anti-Candida activity, demonstrated by the decreased $C$. albicans infection in G. mellonella.

\section{Acknowledgments}

This work was supported by the Fundação de Amparo à Pesquisa do Estado de São Paulo (FAPESP), Brazil (Scholarship 2016/17166-7).

\section{Conflicts of interest}

The authors declare that they have no conflicts of interest.

\section{References}

[1] R.D. Rossoni, J.O Barbosa, F.E de Oliveira, L.D de Oliveira, A.O. Jorge, J.C. Junqueira, Biofilms of Candida albicans serotypes A and B differ in their sensitivity to photodynamic therapy, Lasers Med. Sci. 29 (2014) 1679-1684.

[2] J.C. Sardi, L. Scorzoni, T. Bernardi, A.M. Fusco-Almeida, M.J. Mendes Giannini, Candida species: current epidemiology, pathogenicity, biofilm formation, natural antifungal products and new therapeutic options, J. Med. Microbiol. 62 (2013) $10-24$.

[3] S.G. Whaley, E.L. Berkow, J.M. Rybak, A.T. Nishimoto, K.S. Barker, P.D. Rogers, Azole antifungal resistance in Candida albicans and emerging non-albicans Candida species, Front. Microbiol. 7 (2016) 2173.

[4] J.R. Naglik, S.J. Challacombe, B. Hube, Candida albicans secreted aspartyl proteinases in virulence and pathogenesis, Microbiol. Mol. Biol. Rev. 67 (2003) 400-428 table of contents.

[5] T. Kourkoumpetis, D. Manolakaki, G. Velmahos, Y. Chang, H.B. Alam, M.M. De Moya, et al., Candida infection and colonization among non-trauma emergency surgery patients, Virulence 1 (2010) 359-366.

[6] S.S. Goncalves, A.C. Souza, A. Chowdhary, J.F. Meis, A.L. Colombo, Epidemiology and molecular mechanisms of antifungal resistance in Candida and Aspergillus, Mycoses 59 (2016) 198-219.

[7] A. da Silva Dantas, K.K. Lee, I. Raziunaite, K. Schaefer, J. Wagener, B. Yadav, et al., Cell biology of Candida albicans-host interactions, Curr. Opin. Microbiol. 34 (2016) 111-118.

[8] G.D. Brown, Innate antifungal immunity: the key role of phagocytes, Annu. Rev. Immunol. 29 (2011) 1-21.

[9] C. Bourgeois, O. Majer, I.E. Frohner, L. Tierney, K. Kuchler, Fungal attacks on mammalian hosts: pathogen elimination requires sensing and tasting, Curr. Opin. Microbiol. 13 (2010) 401-408.

[10] P. Badiee, Z. Hashemizadeh, Opportunistic invasive fungal infections: diagnosis \& clinical management, Indian J. Med. Res. 139 (2014) 195-204.

[11] B.J. Kullberg, M.G. Netea, A.G. Vonk, J.W. van der Meer, Modulation of neutrophil function in host defense against disseminated Candida albicans infection in mice, FEMS Immunol. Med. Microbiol. 26 (1999) 299-307.

[12] L. Romani, Immunity to fungal infections, Nat. Rev. Immunol. 11 (2011) 275-288.

[13] S. Kagami, H.L. Rizzo, S.E. Kurtz, L.S. Miller, A. Blauvelt, IL-23 and IL-17A, but not IL-12 and IL-22, are required for optimal skin host defense against Candida albicans, J. Immunol. 185 (2010) 5453-5462.

[14] J.P. Richardson, D.L. Moyes, Adaptive immune responses to Candida albicans infection, Virulence 6 (2015) 327-337.

[15] D.L. Moyes, M. Runglall, C. Murciano, C. Shen, D. Nayar, S. Thavaraj, et al, A biphasic innate immune MAPK response discriminates between the yeast and hyphal forms of Candida albicans in epithelial cells, Cell Host Microbe 8 (2010) 225-235.

[16] M.A. Pfaller, D.J. Diekema, Epidemiology of invasive mycoses in North America, Crit. Rev. Microbiol. 36 (2010) 1-53.

[17] M.L. Cross, Microbes versus microbes: immune signals generated by probiotic lactobacilli and their role in protection against microbial pathogens, FEMS Immunol. Med. Microbiol. 34 (2002) 245-253.

[18] S. Jain, H. Yadav, P.R. Sinha, Stimulation of innate immunity by oral administration of dahi containing probiotic Lactobacillus casei in mice, J. Med. Food 11 (2008) 652-656.

[19] K. Miyazawa, F. He, M. Kawase, A. Kubota, K. Yoda, M. Hiramatsu, Enhancement of immunoregulatory effects of Lactobacillus gasseri TMC0356 by heat treatment and culture medium, Lett. Appl. Microbiol. 53 (2011) 210-216.

[20] H. Dong, I. Rowland, P. Yaqoob, Comparative effects of six probiotic strains on immune function in vitro, Br. J. Nutr. 108 (2012) 459-470.

[21] D.F. Muench, D.J. Kuch, H. Wu, A.A. Begum, S.J. Veit, M.E. Pelletier, et al., Hydrogen peroxide-producing lactobacilli inhibit gonococci in vitro but not during experimental genital tract infection, J. Infect. Dis. 199 (2009) 1369-1378. 
[22] P. Kanmani, R. Satish Kumar, N. Yuvaraj, K.A. Paari, V. Pattukumar, V. Arul, Probiotics and its functionally valuable products-a review, Crit. Rev. Food Sci. Nutr. 53 (2013) 641-658.

[23] H. Morita, F. He, T. Fuse, A.C. Ouwehand, H. Hashimoto, M. Hosoda, et al., Cytokine production by the murine macrophage cell line J774.1 after exposure to lactobacilli, Biosci. Biotechnol. Biochem. 66 (2002) 1963-1966.

[24] S. Tinrat, S. Saraya, M. Traidej Chomnawang, Isolation and characterization of Lactobacillus salivarius MTC 1026 as a potential probiotic, J. Gen. Appl. Microbiol. 57 (2011) 365-378.

[25] E. Oliveira Silva, T. Cruz de Carvalho, I.A. Parshikov, R. Alves dos Santos, F. Silva Emery, N.A. Jacometti Cardoso Furtado, Cytotoxicity of lapachol metabolites produced by probiotics, Lett. Appl. Microbiol. 59 (2014) 108-114.

[26] S.F. Vilela, J.O. Barbosa, R.D. Rossoni, J.D. Santos, M.C. Prata, A.L. Anbinder, et al., Lactobacillus acidophilus ATCC 4356 inhibits biofilm formation by C. albicans and attenuates the experimental candidiasis in Galleria mellonella, Virulence 6 (2015) 29-39.

[27] F.C. Ribeiro, P.P. de Barros, R.D. Rossoni, J.C. Junqueira, A.O. Jorge, Lactobacillus rhamnosus inhibits Candida albicans virulence factors in vitro and modulates immune system in Galleria mellonella, J. Appl. Microbiol. 122 (2017) 201-211.

[28] S. Fedhila, C. Buisson, O. Dussurget, P. Serror, I.J. Glomski, P. Liehl, et al., Comparative analysis of the virulence of invertebrate and mammalian pathogenic bacteria in the oral insect infection model Galleria mellonella, J. Invertebr. Pathol. 103 (2010) 24-29.

[29] J. Chibebe Junior, C.P. Sabino, X. Tan, J.C. Junqueira, Y. Wang, B.B. Fuchs, et al., Selective photoinactivation of Candida albicans in the non-vertebrate host infection model Galleria mellonella, BMC Microbiol. 13 (2013) 217.

[30] M.L. Cross, A. Ganner, D. Teilab, L.M. Fray, Patterns of cytokine induction by gram-positive and gram-negative probiotic bacteria, FEMS Immunol. Med Microbiol. 42 (2004) 173-180.

[31] J.C. Junqueira, S.F. Vilela, R.D. Rossoni, J.O. Barbosa, A.C. Costa, V.M. Rasteiro, et al., Oral colonization by yeasts in HIV-positive patients in Brazil, Rev. Inst. Med. Trop. Sao Paulo 54 (2012) 17-24.

[32] J.C. Junqueira, B.B. Fuchs, M. Muhammed, J.J. Coleman, J.M. Suleiman, S.F. Vilela, et al., Oral Candida albicans isolates from HIV-positive individuals have similar in vitro biofilm-forming ability and pathogenicity as invasive Candida isolates, BMC Microbiol. 11 (2011) 247.

[33] L. Hu, X. Du, T. Li, Y. Song, S. Zai, X. Hu, et al., Genetic and phenotypic characterization of Candida albicans strains isolated from infectious disease patients in Shanghai, J. Med. Microbiol. 64 (2015) 74-83.

[34] A.L. Jorjao, F.E. de Oliveira, M.V. Leao, C.A. Carvalho, A.O. Jorge, L.D. de Oliveira, Live and heat-killed Lactobacillus rhamnosus ATCC 7469 may induce modulatory cytokines profiles on macrophages RAW 264.7, Sci. World J. 2015 (2015) 716749

[35] V. Lievin-Le Moal, A.L. Servin, Anti-infective activities of lactobacillus strains in the human intestinal microbiota: from probiotics to gastrointestinal antiinfectious biotherapeutic agents, Clin. Microbiol. Rev. 27 (2014) 167-199.

[36] I.R. Rajput, L.Y. Li, X. Xin, B.B. Wu, Z.L. Juan, Z.W. Cui, et al., Effect of Saccharomyces boulardii and Bacillus subtilis B10 on intestinal ultrastructure modulation and mucosal immunity development mechanism in broiler chickens, Poult. Sci. 92 (2013) 956-965.

[37] M.P. Diaz-Ropero, R. Martin, S. Sierra, F. Lara-Villoslada, J.M. Rodriguez, J. Xaus, et al., Two Lactobacillus strains, isolated from breast milk, differently modulate the immune response, J. Appl. Microbiol. 102 (2007) 337-343.

[38] G. Weiss, U.E. Schaible, Macrophage defense mechanisms against intracellular bacteria, Immunol. Rev. 264 (2015) 182-203.

[39] S. Carpenter, E.P. Ricci, B.C. Mercier, M.J. Moore, K.A. Fitzgerald, Post-transcriptional regulation of gene expression in innate immunity, Nat. Rev. Immunol. 14 (2014) 361-376.

[40] W. Li, J. Zhou, Y. Xu, Study of the in vitro cytotoxicity testing of medical devices, Biomed. Rep. 3 (2015) 617-620.

[41] S. Segawa, Y. Wakita, H. Hirata, J. Watari, Oral administration of heat-killed Lactobacillus brevis SBC8803 ameliorates alcoholic liver disease in ethanolcontaining diet-fed C57BL/6N mice, Int. J. Food Microbiol. 128 (2008) $371-377$.

[42] S.O. Jang, H.J. Kim, Y.J. Kim, M.J. Kang, J.W. Kwon, J.H. Seo, et al., Asthma prevention by Lactobacillus rhamnosus in a mouse model is associated with CD4(+)CD25(+)Foxp3(+) T cells, Allergy Asthma Immunol. Res. 4 (2012) $150-156$.

[43] A.G. Vonk, M.G. Netea, J.H. van Krieken, Y. Iwakura, J.W. van der Meer, B.J. Kullberg, Endogenous interleukin (IL)-1 alpha and IL-1 beta are crucial for host defense against disseminated candidiasis, J. Infect. Dis. 193 (2006) 1419-1426.

[44] A.G. Hise, J. Tomalka, S. Ganesan, K. Patel, B.A. Hall, G.D. Brown, et al., An essential role for the NLRP3 inflammasome in host defense against the human fungal pathogen Candida albicans, Cell Host Microbe 5 (2009) 487-497.

[45] C.A. Dinarello, Biologic basis for interleukin-1 in disease, Blood 87 (1996) 2095-2147.

[46] K. Ishihara, T. Hirano, IL-6 in autoimmune disease and chronic inflammatory proliferative disease, Cytokine Growth Factor Rev. 13 (2002) 357-368.

[47] K. Hoebe, E. Janssen, B. Beutler, The interface between innate and adaptive immunity, Nat. Immunol. 5 (2004) 971-974.

[48] S.A. Jones, Directing transition from innate to acquired immunity: defining a role for IL-6, J. Immunol. 175 (2005) 3463-3468.

[49] M. Kopf, H. Baumann, G. Freer, M. Freudenberg, M. Lamers, T. Kishimoto, et al., Impaired immune and acute-phase responses in interleukin-6-deficient mice, Nature 368 (1994) 339-342.

[50] Y. Ling, A. Puel, IL-17 and infections, Actas Dermosifiliogr. 105 (Suppl 1) (2014) 34-40.

[51] Z.Z. Yiu, C.E. Griffiths, Interleukin 17-A inhibition in the treatment of psoriasis, Expert Rev. Clin. Immunol. 12 (2016) 1-4.

[52] L. Romani, Immunity to fungal infections, Nat. Rev. Immunol. 4 (2004) 1-23.

[53] W. Vollmer, D. Blanot, M.A. de Pedro, Peptidoglycan structure and architecture, FEMS Microbiol. Rev. 32 (2008) 149-167.

[54] O.J. Park, J.Y. Han, J.E. Baik, J.H. Jeon, S.S. Kang, C.H. Yun, et al., Lipoteichoic acid of Enterococcus faecalis induces the expression of chemokines via TLR2 and PAFR signaling pathways, J. Leukoc. Biol. 94 (2013) 1275-1284.

[55] A.J. Egan, R.M. Cleverley, K. Peters, R.J. Lewis, W. Vollmer, Regulation of bacterial cell wall growth, FEBS J. 284 (2017) 851-867.

[56] C.W. MacPherson, P. Shastri, O. Mathieu, T.A. Tompkins, P. Burguiere, Genome-wide immune modulation of TLR3-mediated inflammation in intestinal epithelial cells differs between single and multi-strain probiotic combination, PLoS One 12 (2017) e0169847.

[57] Y. Shai, Mechanism of the binding, insertion and destabilization of phospholipid bilayer membranes by alpha-helical antimicrobial and cell nonselective membrane-lytic peptides, Biochim. Biophys. Acta 1462 (1999) 55-70.

[58] L. Vertyporokh, P. Taszlow, M. Samorek-Pierog, I. Wojda, Short-term heat shock affects the course of immune response in Galleria mellonella naturally infected with the entomopathogenic fungus Beauveria bassiana, J. Invertebr. Pathol. 130 (2015) 42-51.

[59] G. Wu, Y. Yi, Y. Lv, M. Li, J. Wang, L. Qiu, The lipopolysaccharide (LPS) of Photorhabdus luminescens TT01 can elicit dose- and time-dependent immune priming in Galleria mellonella larvae, J. Invertebr. Pathol. 127 (2015) $63-72$.

[60] L.N. Pham, M.S. Dionne, M. Shirasu-Hiza, D.S. Schneider, A specific primed immune response in Drosophila is dependent on phagocytes, PLoS Pathog. 3 (2007) e26.

[61] O. Roth, B.M. Sadd, P. Schmid-Hempel, J. Kurtz, Strain-specific priming of resistance in the red flour beetle, Tribolium castaneum, Proc. Biol. Sci. 276 (2009) 145-151.

[62] L. Scorzoni, M.P. de Lucas, A.C. Mesa-Arango, A.M. Fusco-Almeida, E. Lozano, M. Cuenca-Estrella, et al., Antifungal efficacy during Candida krusei infection in non-conventional models correlates with the yeast in vitro susceptibility profile, PLoS One 8 (2013) e60047.

[63] R.D. Rossoni, J.O. Barbosa, S.F. Vilela, J.D. dos Santos, P.P. de Barros, M.C. Prata, et al., Competitive interactions between C. albicans, C. glabrata and C. krusei during biofilm formation and development of experimental Candidiasis, PLoS One 10 (2015) e0131700.

[64] F. Sangalli-Leite, L. Scorzoni, E.S.A.C. Alves de Paula, J.F. da Silva, H.C. de Oliveira, J. de Lacorte Singulani, et al., Synergistic effect of pedalitin and amphotericin B against Cryptococcus neoformans by in vitro and in vivo evaluation, Int. J. Antimicrob. Agents 48 (2016) 504-511.

[65] A. Grounta, P. Harizanis, E. Mylonakis, G.J. Nychas, E.Z. Panagou, Investigating the effect of different treatments with lactic acid bacteria on the fate of Listeria monocytogenes and Staphylococcus aureus infection in Galleria mellonella larvae, PLoS One 11 (2016) e0161263.

[66] R.D. Rossoni, B.B. Fuchs, P.P. de Barros, M.D. Velloso, A.O. Jorge, J.C. Junqueira, et al., Lactobacillus paracasei modulates the immune system of Galleria mellonella and protects against Candida albicans infection, PLoS One 12 (2017) e0173332.

[67] G. Cotter, S. Doyle, K. Kavanagh, Development of an insect model for the in vivo pathogenicity testing of yeasts, FEMS Immunol. Med. Microbiol. 27 (2000) 163-169.

[68] D. Bergin, M. Brennan, K. Kavanagh, Fluctuations in haemocyte density and microbial load may be used as indicators of fungal pathogenicity in larvae of Galleria mellonella, Microb. Infect. 5 (2003) 1389-1395. 